\title{
COMPARATIVE PERFORMANCE ANALYSIS OF PHASE DIVISION MULTIPLEXING TECHNIQUES
}

\author{
Musa ÇIBUK ${ }^{* 1}$, Munip GEYLANi² \\ ${ }^{* 1}$ Bitlis Eren University, Department of Computer Engineering, Bitlis/Turkey \\ ${ }^{2}$ Bitlis Eren University, Department of Informatics, Bitlis/Turkey \\ *Corresponding author; E-mail: mcibuk@beu.edu.tr \\ Received: 16 December 2018; Accepted: 29 December 2018
}

The Phase Division Multiplexing (PDM) technique developed for more efficient use of communication resources has recently gain popularity in wireless communication systems. The PDM technique contributes to parallel communication with the multi-channel structure it supports. Unlike the FDM and TDM techniques, which are frequently used in wireless communication, the PDM has two and four channel structures and multiplexes the phase of the signal. It is thought that the performance analyses of the proposed 2 and 4 channel structures will give the researchers an idea of which aspects are superior and where they can be used. In this study, phase division multiplexing structures are analysed and comparative performance analysis are performed and the results are shared.

When PDM structures are analysed, it is seen that 2-channel PDM-BPSK, 2channel PDM-QPSK and 4-channel PDM-BPSK structures have been developed. In order to compare these structures with each other, graphical user interface has been designed by using MATLAB program and constellation diagrams of each structure have been obtained. Simulation studies of these three structures have been performed by using MATLAB / Simulink program. Simulations have been run with the same system parameters and Bit Error Rate (BER) curves have been obtained. Tolerance ranges have been compared over constellation diagrams and bit error rate curves obtained by simulations have been shared.

As a result of the comparing performed in this study; the 2-channel PDMBPSK structure is the simplest and least energy required structure. The 4channel PDM-BPSK structure is advantageous with the number of channels it provides, but it needs the most energy. The 2-channel PDM-QPSK stands out with its symbol / bit rate. It also requires an energy value in the energy range of the other two techniques.

Key words: PDM-BPSK, PDM-QPSK, phase division multiplexing 


\section{Introduction}

The multiplexing process is frequently used in communication systems to deliver communication resources to more than one user and to use communication costs more efficiently. In wireless communication, multiplexing is seen mainly by using FDM (Frequency Division Multiplexing) [1-4], TDM (Time Division Multiplexing) [1, 2, 4, 5] or using these two multiplexing techniques together [1, $5,6]$. While the FDM offers multiple communication channels to the users by dividing the frequency band, the TDM makes multiplexing by allocating the communication path to different users at different times by dividing in time. Apart from these two basic approaches, OFDM (Orthogonal Frequency Division Multiplexing) [7-12], which is a derivative of the FDM technique, and CDM (Code Division Multiplexing) which is a logical multiplexing technique [13-15] is used.

Unlike the general multiplexing techniques used in wireless communication systems, phase division multiplexing (PDM) [16-18] is a new multiplexing technique which multiplexing in the phase of the signal. When the PDM technique is examined, it is seen that there are two- and four-channel structures based on BPSK and QPSK modulations. As it is a new technique, making comparative analyses of these structures of PDM would be useful for researchers.

The rest of the work is organized as follows. In the second chapter, PDM structures are mentioned briefly. The simulation studies prepared for PDM structures are shared and comparative analyzes are made. In Chapter 4, the results obtained are shared.

\section{Phase Division Multiplexing}

Phase Division Multiplexing (PDM) is a new multiplexing technique developed for use in wireless communication systems, which can be used independently or together with multiplexing techniques commonly used [18]. With Phase Division Multiplexing, phase channels are formed by dividing in the signal phase. These phase channels can be assigned separately to users.

The developed PDM has a structure dependent on the type of modulation to be used. The modulation to be used in PDM has a fixed amplitude value such as PSK [19, 20] modulation and is generated by using pairs of signals with a phase difference of $180^{\circ}$ from each other. With the PDM, the signal phase of $360^{\circ}$ is divided by the number of channels desired to obtain. The constellation diagrams are generated according to the number of phase channels, the modulation level and the possible input conditions. Data communication is performed according to these constellation diagrams.

In the structure formed by symmetric partitioning, signals with different phase values are allocated to each channel. The each channel uses these special signals according to its own data. Because these signals emitted from the channels have the same frequency value, they overlap and a single symbol is received by receiver, which is the vector sum of all these symbols. The PDM is based on the computations and calculation of these overlapping signals, at which point overlap or neutralization may occur. In order to avoid such collision or neutralization, a phase channel is formed in two or multiples of two. In addition, increasing the number of phase channels to be created means that the number of symbols will increase. The large number of symbols to be processed means system complexity and cost. For these reasons, Dual-channel PDM-BPSK, Dual-channel PDM-QPSK and Quad-channel PDMBPSK structures have been developed.

In the PDM, there is a third "-" state indicating the non-use status of the channels in addition to the "1" and "0" data in the input state. All possible combinations of 3 different input states in 
PDM-BPSK structure are 3n, with " $\mathrm{n}=$ number of channels". That is, $3 \mathrm{n}$ symbol signals are processed. In PDM-QPSK, there are 5 different possible input states such as "00", "01", "10" "11" and the non-use condition of the channel "--". This means that $5 \mathrm{n}$ symbol signals are processed, which are different from each other as the phase and amplitude to represent all possible input states.

In the Dual-channel PDM-BPSK, the signal phase with $360^{\circ}$ is divided into 4 equal parts and the signals opposite to each other are allocated to the same channel. The channels emit these signals according to the data they want to transmit. The sum of these signals forms the constellation diagrams of the PDM structure since both channels simultaneously emit signals. The Dual-channel PDM-BPSK has 9 different input states and each symbol represents a 2-bit data set. The first bit of each symbol represents the 1 st channel data and the second bit represents the 2 nd channel data. The rec eiver makes sense of the symbol signal according to the tolerance intervals and separates the data of the channels.

The difference of PDM-QPSK from PDM-BPSK structure is to assign 4 signal values to the generated channels. Thus, 2-bit data can be transmitted from each channel in the QPSK structure. In the Dual-channel PDM-QPSK, the signal phase of $360^{\circ}$ is divided into 8 and 4 signals are allocated per channel to each other. In this structure, communication is provided via 4-bit data groups. The first two digits of these data groups represent the data for the first channel and the last two digits represent the data for the second channel. Each symbol in the constellation diagram represents 4-digit data groups consisting of "0", "1" and "-". While all possible combinations of 4-bit data can create 16 different states, 25 different input states are observed due to the non-use of the channel in the Dual-channel PDM-QPSK structure. These 25 different input states are represented by 25 symbol signals each with different amplitude and phase values.

In the Quad-channel PDM-BPSK, the signal phase of $360^{\circ}$ is divided into 8 equal parts. The signal pairs with opposite angles are assigned to the same channel. In the Quad-channel PDM-BPSK structure, the 4-bit data groups belonging to a different channel of each digit are communicated. Each symbol in the constellation diagram represents the 4-digit data group consisting of "0", "1" and "-", and 81 different input states are observed. These 81 different input states are represented by 81 symbol signals, each with different amplitude and phase values. The receiver determines which signal is received by the amplitude and phase value of the signal to be received.

\section{Simulation}

\subsection{Graphical User Interface}

In this study, a graphical user interface (GUI) has been designed to compare PDM techniques with constellation diagrams and tolerance ranges. This GUI generates the constellation diagram according to the desired input values and determines the tolerance intervals. The Quad-channel PDMBPSK constellation diagram and the tolerance interval analysis are shown by means of the GUI designed in Figure 1. 


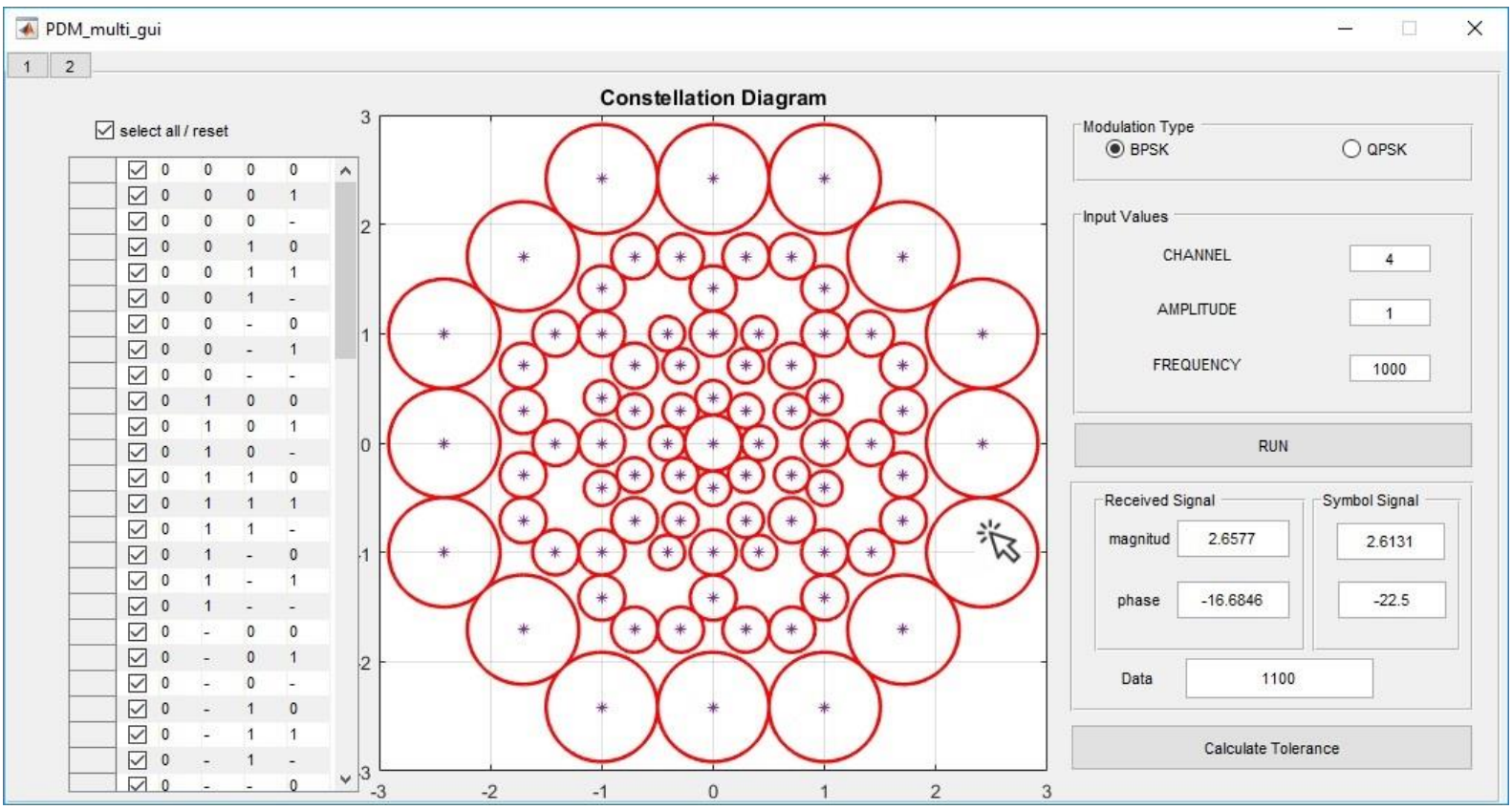

Figure 1. Constellation diagram and tolerance analysis

\subsection{Simulation and Comparison}

In order to test the performance of PDM structures, dual and quad channel PDM modulators and demodulators have been designed in MATLAB / Simulink environment and simulations have been performed by adding noise factor to the transmission medium. Figure 2, Figure 3 and Figure 4 show the Dual-channel PDM-BPSK, Dual-channel PDM-QPSK and Quad-channel PDM-BPSK simulation models respectively. In addition to the use of ready blocks of Simulink program for simulations, special blocks and functions have been developed to represent the study-specific PDM structure.

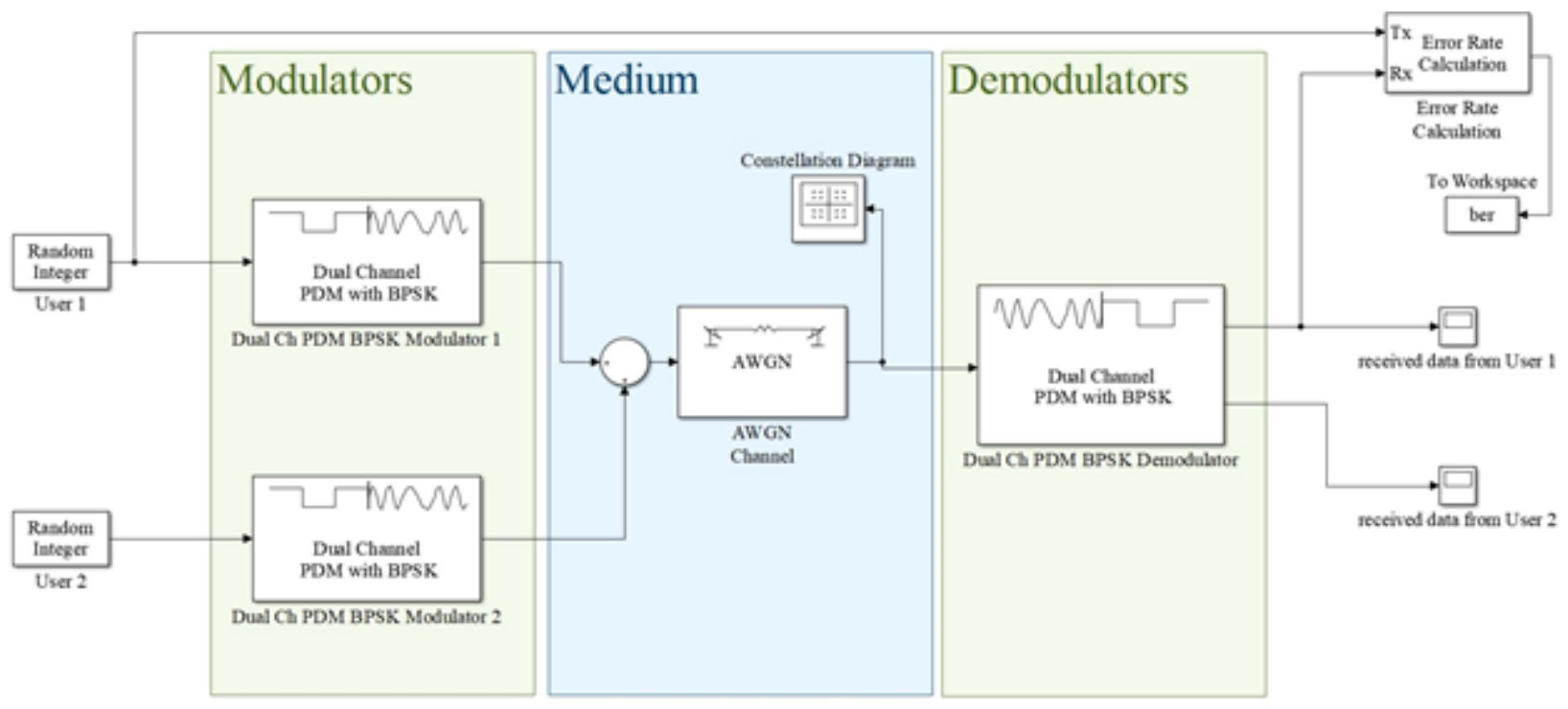

Figure 2. Dual-channel PDM-BPSK simulation 


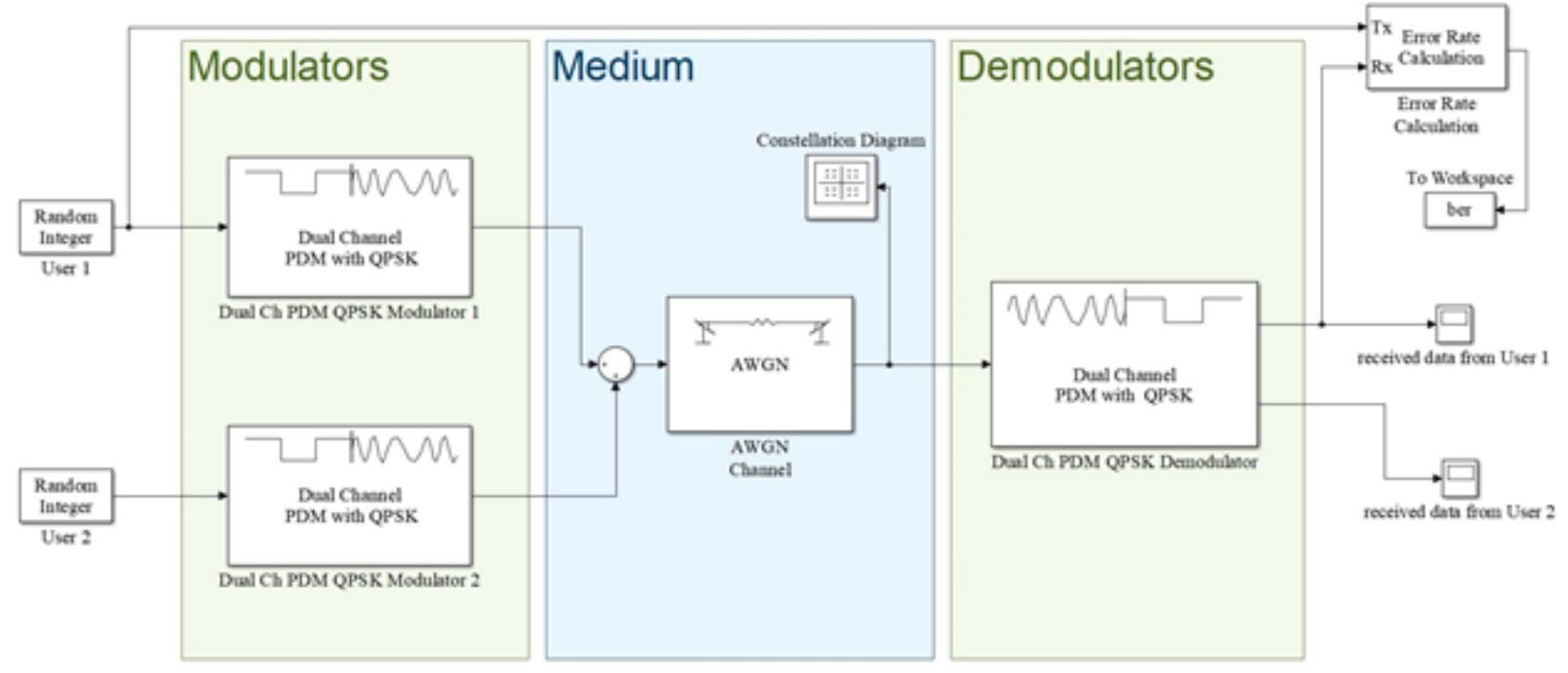

Figure 3. Dual-channel PDM-QPSK simulation

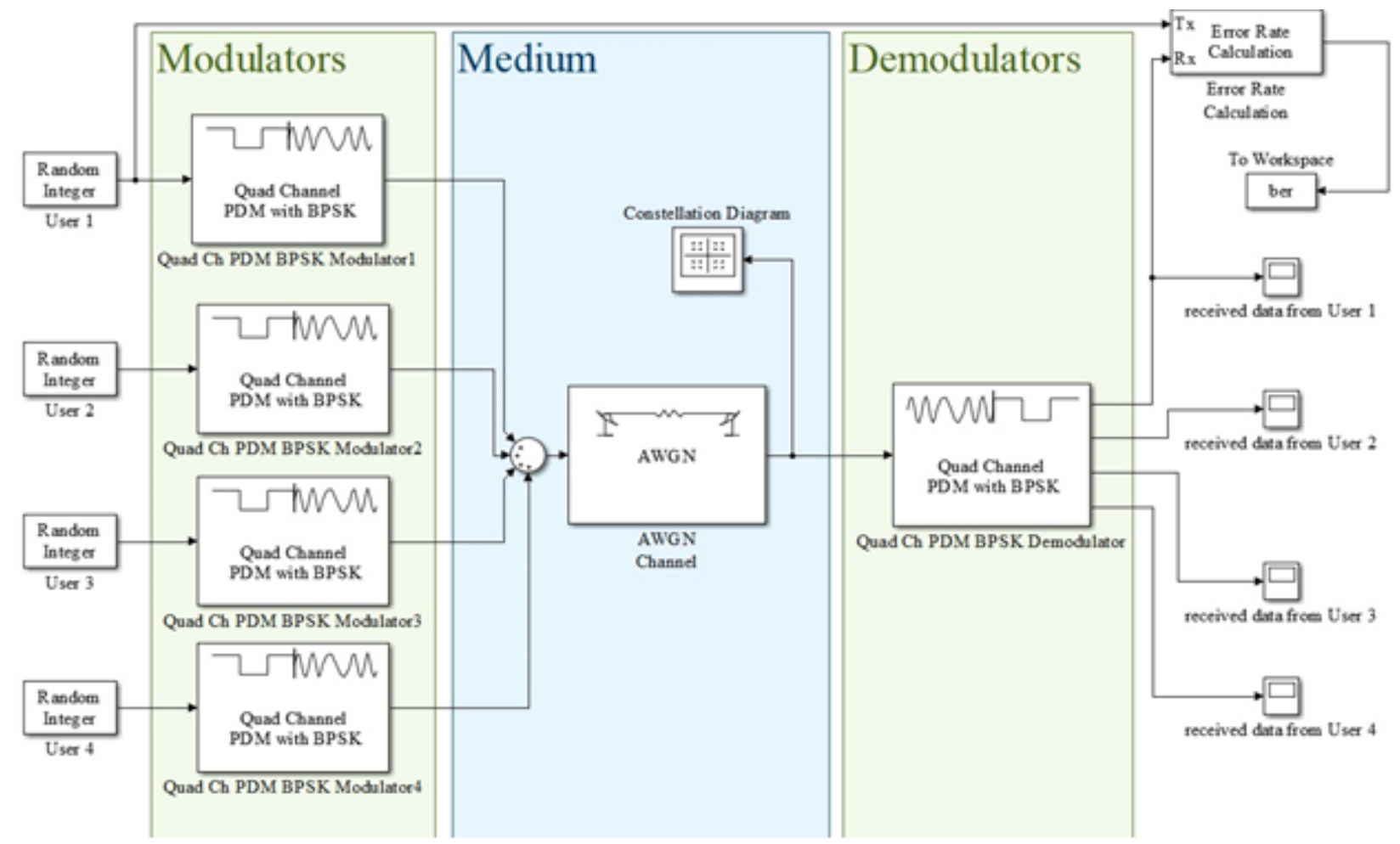

Figure 4. Quad-channel PDM-BPSK Simulation

For " $\mathrm{A}=$ signal amplitude assigned to the channels" and " $\mathrm{f}=$ operating frequency", the PDM simulations have been performed with the parameters given below and the resulting clustering diagrams are given in Figure 5. 


$$
\begin{array}{rll}
\mathrm{A} & : 1 \mathrm{~V} \\
\mathrm{f} & : 100 \mathrm{~Hz} \\
\text { Sample time } & : 1 \mu \mathrm{s} \\
\text { Symbol period } & : 1 \mu \mathrm{s} \\
\text { Run Time } & : 1 \mathrm{~s} \\
\mathrm{E}_{\mathrm{b}} / \mathrm{N}_{\mathrm{o}} & : 10 \mathrm{~dB}, 15 \mathrm{~dB}, 20 \mathrm{~dB}, 24 \mathrm{~dB}
\end{array}
$$
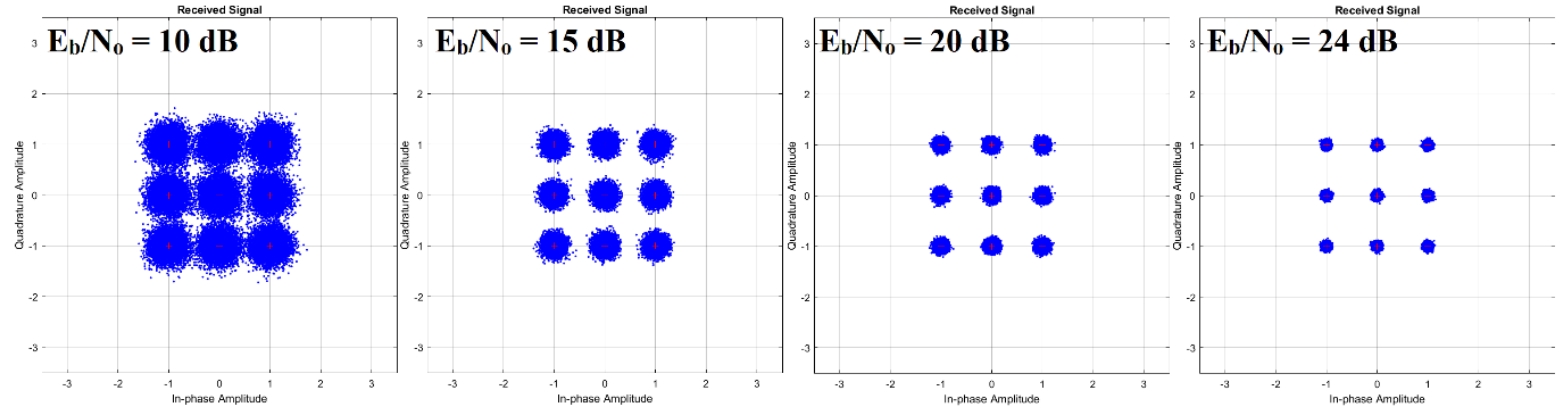

(a) Dual-channel PDM-BPSK
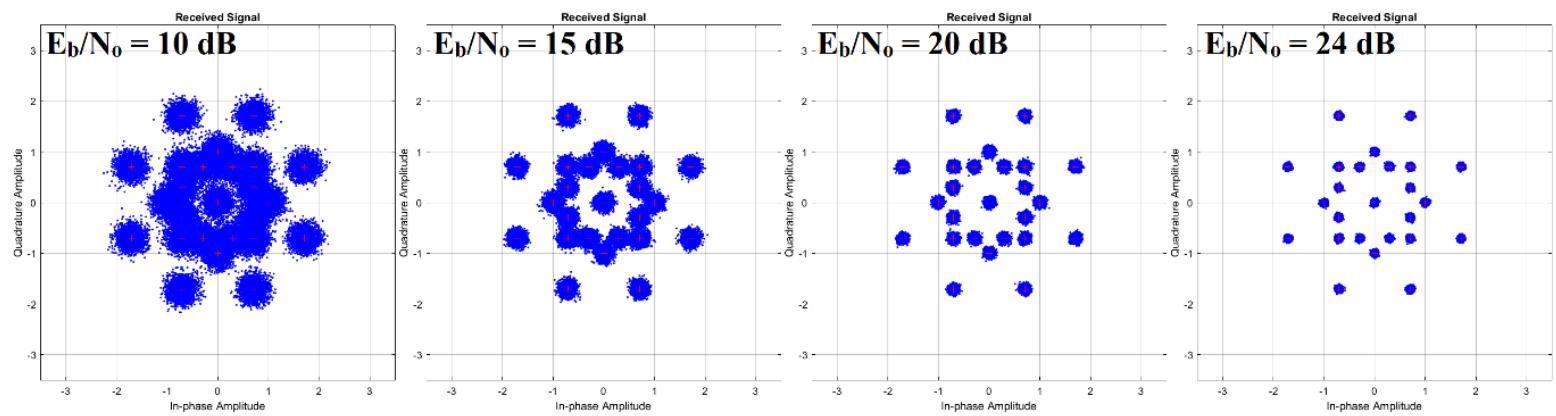

(b) Dual-channel PDM-QPSK
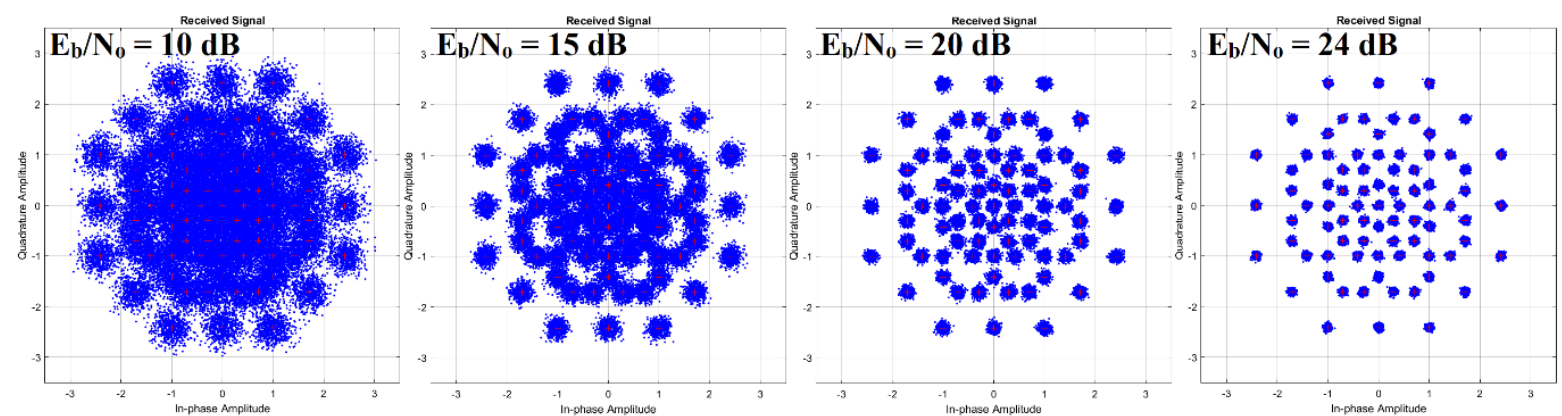

(c) Quad-channel PDM-BPSK

Figure 5. Constellation diagrams of PDM structures for different $E_{b} / N_{o}$ values

As seen in Figure 5, the symbol signals approach each other as the value of $\mathrm{E}_{\mathrm{b}} / \mathrm{N}_{\mathrm{o}}$ decreases. In this case, the desired performance under a certain value can not be achieved because of the overlap. Symbol signals received for $\mathrm{E}_{\mathrm{b}} / \mathrm{N}_{\mathrm{o}}=15 \mathrm{~dB}$ for Daul-channel PDM-BPSK, $\mathrm{E}_{\mathrm{b}} / \mathrm{N}_{\mathrm{o}}=20 \mathrm{~dB}$ for Dualchannel PDM-QPSK and $\mathrm{Eb} / \mathrm{N}_{\mathrm{o}}=24 \mathrm{~dB}$ for Quad-channel PDM-BPSK are severely separated from each other and grouped within specified tolerance limits. 
The Bit Error Rate Analysis Tool (BERTool) tool has been used to calculate BER values corresponding to different $\mathrm{E}_{\mathrm{b}} / \mathrm{N}_{\mathrm{o}}$ values with the parameter values specified in the simulations. BER curves for all three PDM structures are shown in Figure 6.

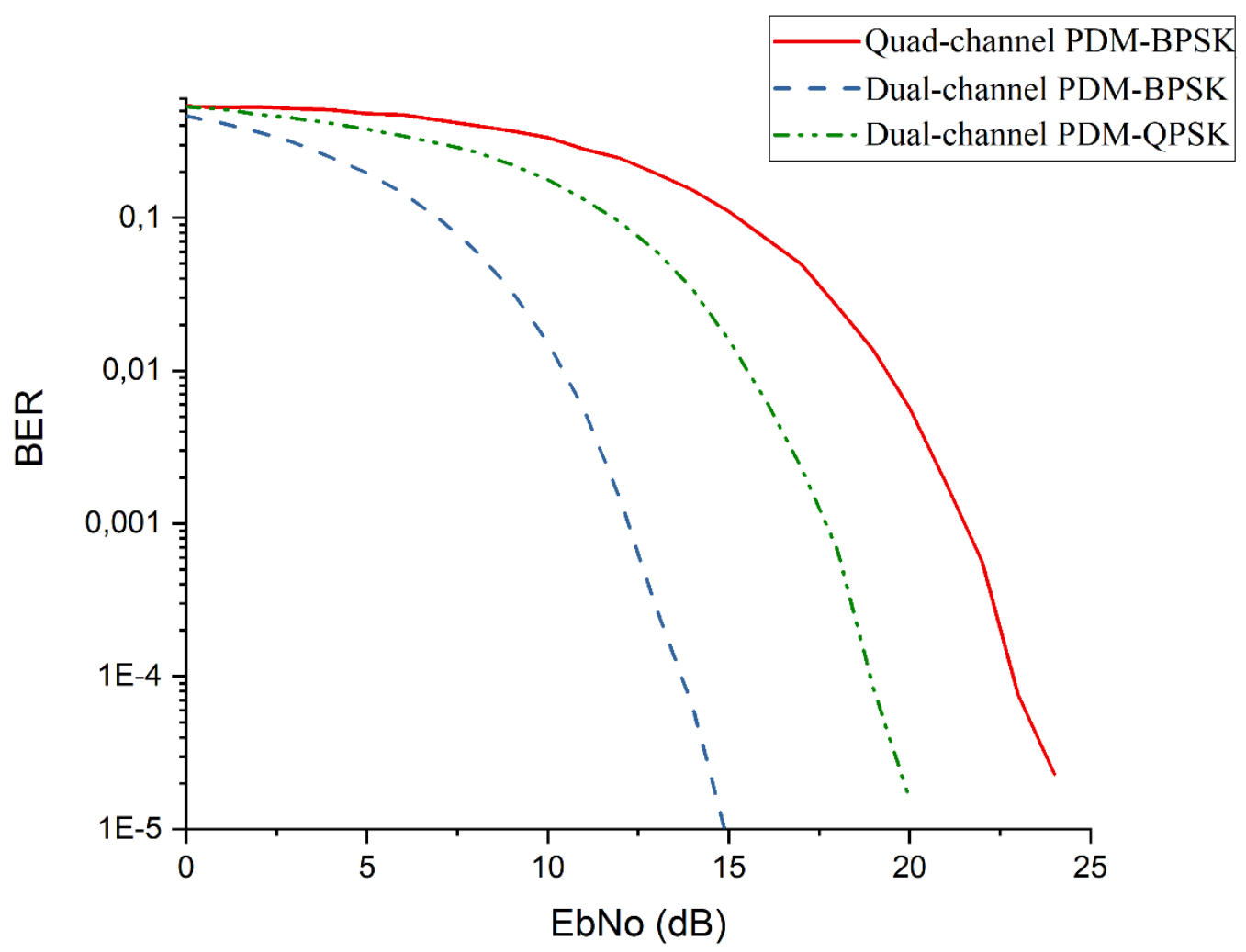

Figure 6. BER performance comparison of PDM structures

As it seen from the graph; as the number of channels increases, the required $\mathrm{E}_{b} / \mathrm{N}_{\mathrm{o}}$ is increasing. Also when higher level modulation is used in PDM, it is seen that the required $\mathrm{E}_{\mathrm{b}} / \mathrm{N}_{\mathrm{o}}$ value increases. The reason for this is that when the number of channels increases or the modulation level is increased, the symbol signals that come up are close to each other and their tolerances decrease. The comparison data obtained in the study are summarized in Table 1.

Table1. Pdm Techniques Comparison

\begin{tabular}{|c|c|c|c|c|c|}
\hline & Bit/Symbol Rate & Modulation & Symbol & $\begin{array}{c}\mathbf{E}_{b} / \mathbf{N}_{\mathbf{~ d B}} \\
\left(\mathbf{B E R}=\mathbf{1 0}^{-5}\right)\end{array}$ & Channel \\
\hline $\begin{array}{c}\text { Dual -channel } \\
\text { PDM-BPSK }\end{array}$ & 2 & BPSK & 9 & 15 & 2 \\
\hline $\begin{array}{c}\text { Dual-channel } \\
\text { PDM-QPSK }\end{array}$ & 4 & QPSK & 25 & 20 & 2 \\
\hline $\begin{array}{c}\text { Quad-channel } \\
\text { PDM-BPSK }\end{array}$ & 4 & BPSK & 81 & 24 & 4 \\
\hline
\end{tabular}




\section{Conclusion}

In this study, a comparative analysis of PDM, a new multiplexing technique, has been introduced. For this purpose, a graphical user interface has been designed by using MATLAB environmentand clustering diagrams of each structure are obtained. Using the MATLAB / Simulink environment, the simulations of these three structures are made and the results of the simulations operated in the same parameters are shared. Tolerance ranges are compared over clustering diagrams and bit error rate curves obtained by simulations are shared.

As seen in the study two independent channels are obtained in Dual-channel PDM-BPSK and Dual-channel PDM-QPSK techniques and 4 independent channels are obtained by Quad-channel PDMBPSK technique. The Dual-channel PDM-BPSK technique requires 25 percent less energy than the Dual-channel PDM-QPSK technique and the Quad-channel PDM-BPSK technique needs 20 percent more energy than the Dual-channel PDM-QPSK technique. In Dual-channel PDM-BPSK and Quadchannel PDM-BPSK techniques, 1 bit can be transmitted from channels simultaneously, while Dualchannel PDM-QPSK technique can be transmitted by 2 bits simultaneously.

As a result, in this study; with the simple structure of the Dual-channel PDM-BPSK, it is seen to be the least energy-require technique. The small number of symbols processed in this case has a great contribution. In this technique, each channel can transmit 1 bit data at the same time. The Quad-channel PDM-BPSK provides the highest number of channels and is also disadvantageous in terms of energy demand. This is the result of the processing of 81 symbols in the Quad-channel PDM-BPSK structure. In this technique, channels can transmit 1 bit data at the same time. Finally, the energy requirement in the Dual-channel PDM-QPSK structure has a value between the two other techniques. In addition, since the channels can transmit 2-bit data at the same time, it can be seen that it comes forward with a symbol / bit rate.

\section{References}

[1] White, C., Data Communications and Computer Networks: A Business User's Approach, Course Technology Cengage Learning, USA, 2015

[2] Ryan, M. J., Frater, M., Communications and Information Systems, Argos Press P/L, Canberra, Australia, 2002

[3] Patranabis, D., Telemetry Principles, Tata McGraw Hill Education, New Delhi, India, 1999

[4] Schiller, J. H., Mobile Communications, Pearson Education, London, England, 2003

[5] Stallings, W., Data and Computer Communications eight edition, Pearson Education Inc., New Jersey, USA, 2007

[6] Çıbuk, M., Protocol Design And Implementing for Web Based Bio-Telemetry Applications via Wimax/Ieee802.16 Networks, Ph. D. thesis, Firat University, Elazı̆̆, Türkiye, 2009

[7] Li, Y. G., Stuber, G., Orthogonal frequency division multiplexing for wireless communications, Springer Science \& Business Media., Berlin, Germany, 2006

[8] Al-Tamimi, A. K., Exponential Effective Signal to Noise Ratio Mapping (EESM) Computation for Wimax Physical Layer, Master of Science Thesis, Washington University, Washington, 2007

[9] Prasad, R., OFDM for Wireless Communications Systems, Artech House, Boston\&London, England, 2004

[10] Kaur, S., Bharti, G., Orthogonal Frequency Division Multiplexing in Wireless Communication Systems : A Review, Int. J. Adv. Res. Comput. Eng. Technol., 1 (2012), 3, pp. 125-129 
[11] Rashmi, R., Sarala, S. M., OFDM : Modulation Technique for Wireless Communication, Int. J. Innov. Res. Adv. Eng., 1 (2014), 1, pp. 1-5

[12] Arrano, H. F., Azurdia-Meza, C. A., OFDM: Today and in the Future of Next Generation Wireless Communications, 2016 IEEE Central America and Panama Student Conference (CONESCAPAN), Guatemala City, Guatemala, 2016, Vol. 1, pp. 1-6

[13] Hoffmann, C., Code Division Multiplexing, Ph. D thesis, Massachusetts Institu of Technology, Massachusetts, USA, 2004

[14] Jordan, R.., Abdallah, C. T., Wireless. Communications and An Overview, IEEE Antennas Propag. Mag., 44, (2002), 1, pp. 185-193

[15] Fan, P., Multiple Access Technologies for Next Generation Mobile Communications, ITS Telecommunications Proceedings, 2006 6th International Conference, Chengdu, China, 2006, Vol. 1, pp. 10-11

[16] Geylani, M., Çıbuk, M., Multichannel Approach with Phase Based Multiplexing in Wireless Communication and Its Feasibility, International Conference on Multidisciplinary, Science, Engineering and Technology (IMESET'17 Bitlis), Bitlis, Turkey, 2017, Vol. 1, p. 267

[17] Geylani, M., Çıbuk, M., Phase Division Multiplexing with 4-level PSK Modulation and Simulation, IDAP 2018: International Conference on Artificial Intelligence and Data Processing'18, Malatya, Türkiye, 2018

[18] Geylani, M., Improving a New Time-Independent Transmission Technique Using Hybrid Multiplexing Methods in Wireless Sensor Network, Master Thesis, Bitlis Eren University, Bitlis, Türkiye, 2018

[19] Tomasi, W., Atakay, M., Elektronik İletişim Teknikleri, MEB Yayınları, Ankara, Türkiye, 2002

[20] Sharma, D. K., et al., Analog \& Digital Modulation Techniques: An Overview, Tech. Int. J. Comput. Sci. Commun. Technol., 3, (2018), 1, pp. 551-561 